\title{
Cheyne-Stokes Respiration Detection Method for Newborns with Apnea
}

\author{
Taiga Niimi ${ }^{\dagger}$, Yushi Itoh ${ }^{\dagger \dagger}$, Michiya Natori ${ }^{\dagger \dagger}$ and Yoshimitsu Aoki ${ }^{\dagger}$ (member)
}

\begin{abstract}
Cheyne-Stokes respiration (CSR) has a high prevalence among newborns, especially among preterm babies. Although doctors generally recognize the phenomenon, they are not able to assess the severity of CSR in individual infants. CSR is characterized by cyclical weakening and strengthening of respirations with apnea. In this study, we developed an apnea detection method, and a CSR detection method using detected apneic events. We detected apnea using two features of respiratory waveforms. The first feature is frequency information calculated from wavelet coefficients. The second is information based on the shape of the waveform. In our CSR detection method, we used a spurious periodicity feature to determine CSR sections. The waveform is calculated by a respiratory monitoring system that uses a fiber-grating vision sensor to measure the vertical motion of the infant's thoracic and abdominal regions during respiration. Our method is effective at detecting apnea (sensitivity: $94.3 \%$, specificity: $99.7 \%$ ).
\end{abstract}

Keywords: Newborn, Preterm, Apnea, Cheyne-Stokes respiration, Wavelet, FG vision sensor.

\section{Introduction}

In recent years, the rate of low birth weight has been increasing. Low birth weight (under $2500 \mathrm{~g}$ ) may be caused by preterm delivery or agenesis in utero. The mortality rate of low birth weight babies is high because their respiratory and other body functions are immature. Prevalent respiratory diseases in preterm babies include neonatal respiratory distress syndrome, episodes of apnea, and sudden infant death syndrome (SIDS). The incidence of these diseases increases with lower gestational age. Respiratory monitoring of newborns is crucial because it enables early diagnosis and prevention of disease. Early discovery of respiratory disorders and estimation of the state of respiratory function allows proper medical treatment at an early stage. For this reason, we believe that respiratory monitoring saves the lives of newborns.

In neonatal intensive care units, neonatal respiration is monitored with two contact-type sensors. The first sensor is a cardio-respiratory monitor that measures respiratory rate and heart rate ${ }^{1)}$. This sensor measures changes in impedance when slight electrical current is

\footnotetext{
Received Fabruary 28, 2013; Revised June 22, 2013; Accepted July 31, 2013 $\dagger$ Keio University

(Hiyoshi 3-14-1, Kohoku-ku, Yokohama-shi, Kanagawa, Japan)

$\dagger$ National Center for Child Health and Development

(2-10-1 Okura Setagaya-ku Tokyo, Japan)
}

applied to electrodes attached to the infant's body, and it detects chest and abdominal movements. The second sensor is a pulse oximeter, which indirectly measures blood oxygen saturation $(\mathrm{SpO} 2)^{2)}$. It has a pair of small light-emitting diodes facing a photodiode and is most commonly applied to a fingertip or an earlobe. Because absorption of red and infrared light differs between oxyhemoglobin and its deoxygenated form, $\mathrm{SpO} 2$ can be calculated from the ratio of the absorption of these wavelengths. However, contact-type sensors may cause irritation to the newborn's sensitive skin. In addition, they may hinder medical treatments and often detach because of treatments or the baby's movements. Furthermore, the monitors' detection accuracy varies with the location of sensor attachment.

Cheyne-Stokes respiration (CSR) is an abnormal breathing pattern characterized by gradual cyclical alternations between apnea and normal breaths. CSR is a severe state of periodic breathing caused by abnormal function of the brain region that controls respiration. People with CSR do not respond quickly enough to changes in blood levels of oxygen and carbon dioxide.

CSR has a high prevalence among newborns, especially among preterm newborns, whose central nervous systems are immature. CSR is triggered by hypoxia associated with hypoventilation ${ }^{3)}$, because respiratory control is unstable when the central nervous 
system is immature. CSR is closely associated with respiratory function immaturity in preterm newborns ${ }^{4}$. For this reason, estimating the severity of CSR could provide information about respiratory function maturity. Furthermore, CSR analysis has the potential to allow early detection of diseases (e.g. excessive periodic breathing is seen in infants with near-miss $\operatorname{SIDS}^{5) 6)}$ ) and to contribute to an understanding of the respiratory physiology of newborns. However, CSR in newborns has not been broadly studied, especially in the signalprocessing field, because of the disadvantages of respiratory contact-type sensors. These sensors have prevented researchers from gaining sufficient data, for fear of imposing a burden on preterm babies and babies with CSR. Moreover, as mentioned above, the detection accuracy of respiratory contact-type sensors is variable.

To solve these problems, we developed a respiratory monitoring system for newborns using a fiber-grating (FG) vision sensor ${ }^{7)}$. The FG vision sensor is an active stereo $3 \mathrm{D}$ sensor that allows noncontact $3 \mathrm{D}$ measurements ${ }^{8)}$. This system can calculate more accurate respiratory waveforms than traditional medical sensors. Using this system, we developed a novel method to estimate the severity of CSR.

Previous work using only the respiratory waveform shape or only frequency information is not sufficiently robust for apnea detection in newborns. Moreover, using a single feature or detection function results in large trade-offs between detection accuracy and threshold sensitivity.

In this paper, we propose methods to detect apnea and CSR. Duration of apnea in newborns is shorter than in adults and neonatal respiratory waveforms tend to be distorted. To increase the robustness of detection accuracy, we adopted three different apnea detection functions using features of respiratory frequency and waveform shape information. The use of two different features improved detection accuracy. Our method also reduces false negatives by employing three different functions to ensure that at least one of the three detects apnea, even when thresholds are strict to avoid false positives.

We also propose a method to detect CSR associated with apneic events found by our apnea detection method. To detect CSR sections in the waveform time axis, our method uses frequency information calculated from detected apneas. We aimed to avoid using frequency information from the envelope curve of the waveforms, considering the distorted waveforms of neonates.

This paper is organized as follows. In Section 2, we discuss related research. Section 3 describes our respiratory monitoring system with the $\mathrm{FG}$ vision sensor. Section 4 presents our apnea detection method. In Section 5, we explain our CSR detection method. Finally, we present our conclusions and propose future work.

Our apnea detection method and CSR detection method have four main contributions: apnea analysis, CSR detection, estimation of CSR severity, and improved understanding of the respiratory physiology of newborns.

\section{Related Studies}

Previous studies using waveform analysis have described classification methods for CSR associated with cardiac failure ${ }^{9) 10}$. One study classified CSR by analyzing respiratory frequency using the envelope curve of the waveform. Although the classification accuracy of this method exceeded $80 \%$, it was proposed for adults, whose waveforms are more stable than those of newborns. The respiratory state of newborns changes frequently in a short time, which distorts the envelope curve. Furthermore, the cycle of apneic events varies among patients and within individual patients. It is problematic to detect CSR in newborns based on the waveform envelope. We focused instead on apnea and developed an apnea detection method, which helped us develop CSR detection.

Apnea is commonly seen in preterm newborns. Apnea is considered a clinical event if it lasts for more than 20 seconds or if it lasts for more than 10 seconds and is accompanied by either bradycardia ( $<80$ beats per minute) or oxygen desaturation (below $80 \%)^{11}$. In these cases, apnea requires prompt medical attention.

Apnea can be detected using polysomnography (PSG) and artificial neural networks (ANNs). Polysomnography uses a multichannel recording instrument with contact-type sensors to record a subject's biophysical changes during sleep $^{12)}$. This method uses PSG signals to detect apnea of more than 10 seconds' duration to diagnose sleep apnea syndrome ${ }^{13)-15)}$. The sampling rate of PSG signals is usually more than $100 \mathrm{~Hz}$, and these methods downsample the signal rates and apply windowing for ANN analysis. However, even after adjustment, the PSG signal (more than $30 \mathrm{~Hz}$ ) has more information than the respiratory waveforms of our system (about $8 \mathrm{~Hz}$ ). Therefore, we cannot apply these methods to our system. 
The focus of the traditional apnea detection methods described above is different from our focus. We are interested not only in clinical apnea but also in apnea lasting less than 10 seconds in newborns. CSR in infants involves not only apneic events lasting longer than 10 seconds but also shorter duration events of 1.5-10 seconds. Respiratory analysis of shorter apneic events contributes to understanding the respiratory physiology of newborns.

Another method of apnea detection depends on standard deviation ${ }^{16)}$. This method uses chest impedance (CI) signals. It first removes the heart rate signal component of the CI signal that appears in apnea sections by resampling the CI signal with an equal number of steps in each R-R interval (filtered CI). A histogram of standard deviation is made at every quarter second for a centered 2-second interval. Next, two distributions corresponding to the deviation histogram of apnea sections and respiratory sections are calculated. Finally, an apnea probability value is calculated from the values of these distributions, corresponding to the calculated standard deviation. Although the accuracy of this method is more than $90 \%$, it ignores all apneic events less than 2 seconds and also events less than 5 seconds unless the event is within 5 seconds of another event. We define apnea as respirations having a wave height less than $0.1 \mathrm{~mm}$ for more than 1.5 seconds, considering the physiological effects explained below. It is difficult to set parameters of the standard deviation method to detect apneic events with wave heights as specified in our definition.

Recent studies on apnea detection in adults have utilized frequency energy calculated by fast Fourier transformation $\left(\right.$ FFT) ${ }^{17)}$ or waveform shape ${ }^{18)}$. Fast Fourier transformation needs a fixed long-duration window to extract accurate frequency information from the waveform. However, the sampling rate of the monitoring system we used is $8 \mathrm{~Hz}$ and the length of apnea we defined for a newborn is as short as 1.5 seconds. Therefore, this method designed for adults is not appropriate for newborns. Moreover, the waveform can be distorted when respiration is unstable. The results of the waveform shape-based method tend to depend on the state of respiration.

Using information from the shape of the waveform or frequency information alone does not achieve adequate robustness for apnea detection in newborns. Moreover, using only one feature or one detection function results in a large trade-off between detection accuracy and threshold sensitivity.

\section{Respiratory Monitoring System}

\subsection{System Description}

The respiratory monitoring system is composed of an FG vision sensor positioned above the newborn and an operational component that calculates the respiratory waveform (Fig. 1(a)). The FG vision sensor ${ }^{8)}$ includes a spot projector and an infrared charge-coupled device (CCD) camera. The spot projector is composed of a diffraction grating of an optical fiber (fiber grating) and an infrared laser. When a laser vertically enters the fiber grating, the spot is irradiated on the screen net, similar to a square lattice. The spot image is obtained by taking a picture of the screen with the infrared CCD camera (Fig. 1(b)). When the depth of the spot irradiation position changes, the coordinate value of the spot changes in a constant direction. It is then possible to calculate the displacement vector of the spot irradiation position from the displacement vector of the spot on the twodimensional (2D) CCD array. With this information, the system calculates the $3 \mathrm{D}$ displacement vector of the spot irradiating the thoracoabdominal region and calculates the respiratory waveform ${ }^{7)}$.

\subsection{Spot Displacement Vector Calculation}

To detect thoracoabdominal movement with respiration, the system calculates the displacement vector of each spot. Fig. 1c shows the optical layout of the FG vision sensor. Parameter $h$ is the distance between the screen and the lens. Parameter $l$ is the focal point distance of the CCD camera. Parameter $d$ is the base length, which is the distance between the center of the lens and the fiber grating. Parameter $\delta$ is the displacement vector of the spot on the 2D CCD array between frames. Calculation of the displacement vector
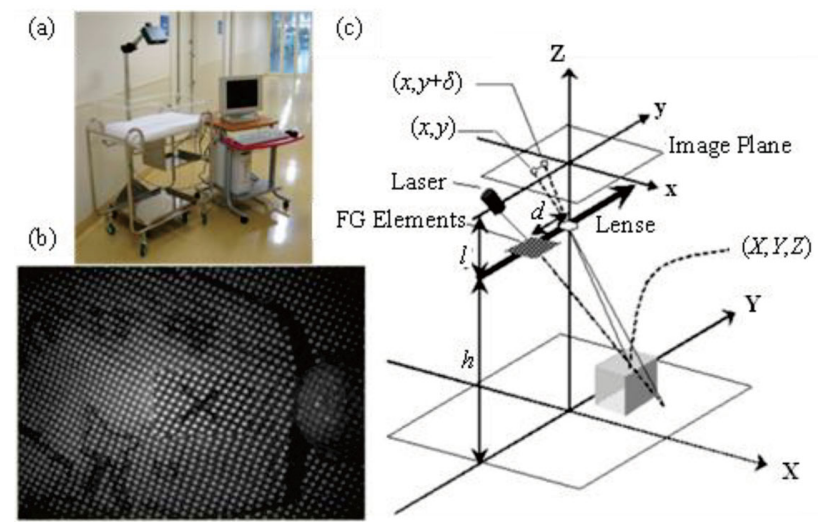

Fig.1 (a) System set-up; (b) Spot image; (c) Optical layout of FG vision sensor. 
of each spot is based on optical triangulation, as shown in Equations (1), (2), and (3).

$$
\begin{aligned}
& d Z=h 2 \delta /(d l+h \delta), \\
& d X=x(h-d Z) / I, \\
& d Y=(y+\delta)(h-d Z) / I,
\end{aligned}
$$

\subsection{Respiratory Waveform}

The respiratory waveform is calculated using the $z$ axial displacement vector. The system calculated the average value of the vectors in the thoracoabdominal region ${ }^{7)}$. This average value is the velocity of the thoracoabdominal region between frames. The sum of all velocities obtained in each frame is the thoracoabdominal height. This system convolves the waveform with a bandpass filter whose frequency scale is $0.17-2.0 \mathrm{~Hz}$, which is the respiratory frequency band of a newborn.

It was found that the system can accurately detect moves (waveform height $=0.5,0.25$ and a respiratory rate $(R R)=30,60,90,120)$ of $Z$-stage which is a positioning stage which moves on a scale of $1 / 1,000$ millimeters in the $z$-axial direction ${ }^{7)}$. The respiratory amplitude range of newborn is around 0.5 millimeters. The usual respiratory rate of newborn for one minute is around 35 times. On the other hand, the respiratory amplitude range of preterm is unknown and its respiratory rate can exceed 100 times because the most newborns which we monitored by using our system get better regarding the degree of maturity. It is the reason that why not only 0.5 but also 0.25 of waveform height are selected.

Fig. 2 shows results from 100 seconds of the respiratory waveforms of an adult and a newborn, calculated by the described system. The adult respiratory rate is about $15-20$ breaths per minute, whereas that of newborns is about 30-120 breaths per minute. The range of respiratory waveform height of a

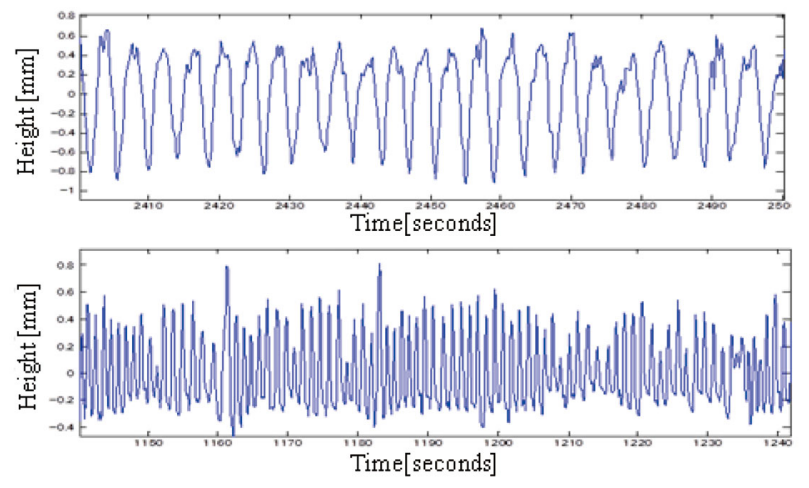

Fig.2 Respiratory waveforms. Top: adult; bottom: newborn. newborn is $0.1-1.0 \mathrm{~mm}$, with waveform instability because of varied respiration within a short time.

The system monitors neonatal respiration and detects apnea in real time. It sets off an alarm when the detected apneic period is longer than 20 seconds because longer-duration apnea could result in infant death.

The system also detects non-respiratory movements of newborns. In general, the system can accurately detect respiratory movement because the waveform is calculated from z-axial displacement vectors of the spots in thoracoabdominal region and the values of waveform are less influenced by a little movement of limbs. Furthermore, the preterm newborns rarely initiate body movements and pose changes. On the other hand, if a newborn moves or changes its pose, the average value of the displacement vectors of the thoracoabdominal region becomes much larger than that during breathing movements. We empirically regard the section where wave height more than $1.0 \mathrm{~mm}$ lasts more than 10 seconds as body motion period and detect abnormal respirations (apnea and CSR) in the respiratory waveform except the body motion sections. It is easy to distinguish body motion from respiratory waveforms although the system cannot calculate accurate waveform in the period of body motion.

\section{Apnea Detection}

A doctor at the National Center for Child Health and Development (NCCD) with whom we collaborate watched the thoracoabdominal motion of newborns during respiration while simultaneously watching a signal from our system and from the monitoring system used at the NCCD. In consultation with this doctor, we defined apnea in newborns as respirations having a wave height less than $0.1 \mathrm{~mm}$ for more than 1.5 seconds.

\subsection{Proposed Apnea Detection Method}

Our method employs three features containing both frequency and waveform shape information for robustness of detection accuracy in unstable respiration. One feature uses frequency information calculated from wavelet coefficients. The others are related to the shape of the respiratory waveform. Our method has three different functions using these features to reduce the trade-offs between detection accuracy and sensitivity threshold. At least one of the three functions can detect apnea even with high thresholds to avoid false positives. Our method combines the results from these three functions to generate the "apnea label".

Wavelet transform (WT) is a time-frequency analysis 
that determines the proper time and frequency resolution. Equation (4) shows the wavelet function. The frequency scale of the wavelet can be altered by changing the scale parameter $a$, and the width of the wavelet can be changed to the optimal width for the band of frequencies at the same time. Equation (5) shows how WT convolves the waveform with the wavelet. The result of the transform is called the "wavelet coefficient",

$$
\begin{aligned}
& \psi_{a, b}(t)=\psi\left(\frac{t-b}{a}\right) / \sqrt{a}, \\
& w_{a, b}=\int_{-\infty}^{\infty} \psi(t) \psi_{a, b}(t) d t,
\end{aligned}
$$

where $a$ is the scale parameter, $b$ is the time parameter, and $x(t)$ is the respiratory waveform. Equation (6) shows orthonormal wavelet function for discrete wavelet transform (DWT). The parameters of an orthonormal wavelet are proportional to a factor of 2 :

$$
2^{-j / 2} \psi\left(2^{-j} t-k\right)=2^{-j 2} \psi\left(2^{-j 2}-k\right)
$$

where $a=2^{j}, b=2^{j} k$, and $j$ is the resolution level of octave decomposition. If the value $j$ increases, the wavelet frequency scale makes the shift to a lower frequency.

To obtain frequency information, we selected $9 / 7$ wavelet, which is used in lifting-based DWT scheme wavelet transform. The main feature of the lifting-based DWT scheme is to break up the high-pass and low-pass wavelet into a sequence of upper and lower triangular matrices, and convert the filter implementation into banded matrix multiplications ${ }^{19)}$. This scheme often requires few computations compared to the convolution based DWT. However, we only require the symmetrical waveform of $9 / 7$ wavelet and the similarity of its shape to a respiratory waveform, because we want to match changes in the height of the respiratory waveform and in the wavelet coefficient temporally.

We employed wavelet resolution levels 2, 3, and 4 of octave decomposition because the sampling rate of the waveform was $8 \mathrm{~Hz}$, and the breathing cycle of newborns is about 0.5-2 seconds. The frequency ranges of resolution levels 2,3 , and 4 are $1-2 \mathrm{~Hz}, 0.5-1 \mathrm{~Hz}$, and 0.25-0.5 Hz, respectively. We calculated wavelet coefficients with equation (5), from the waveform before applying the band-pass filter, for each resolution level at every point. We squared these wavelet coefficients for each time point. The sum of these values was reassigned to a new sequence (Fig. 3). We call this feature "wavelet

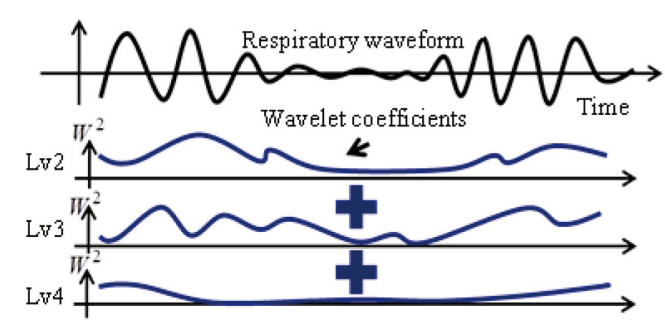

Fig.3 Calculation of wavelet energy.

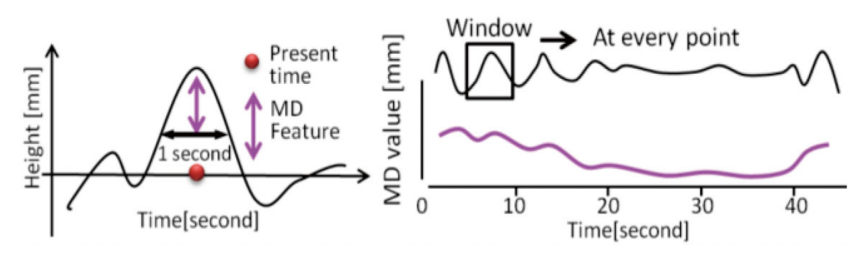

Fig.4 Illustration of the MD feature.

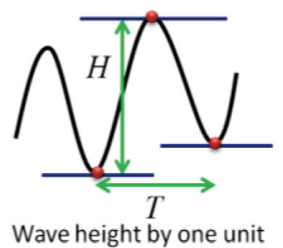

Fig.5 Illustration of the SI feature.

energy", and it represents respiratory frequency energy.

To analyze information from the shape of the respiratory waveform, we used the maximum displacement (MD) feature and shape information (SI). The MD feature is calculated by sampling the maximum displacement of the waveform, before applying the band-pass filter, in 1.0second intervals centered on the present time (Fig. 4). The value of MD increases in respiration sections and decreases in apnea sections. Fig. 5 illustrates the SI feature. We first detect the extrema of the respiratory waveform, which is the waveform after applying the band-pass filter because the waveform not applied the filter has many extrema. The waveform between the left and right minima is regarded as one unit shape. Next, we calculate the time duration $T$ and wave height $H$ of one unit shape.

We employed three functions to detect apnea. The first function is based on frequency information.

Wavelet Energy < 0.03,

$\mathrm{MD}<0.1225$.

The main element of this function is wavelet energy. Wavelet can extract frequency information of waveform in its time window. Therefore it can stably extract usable information from even unstable waveform. 


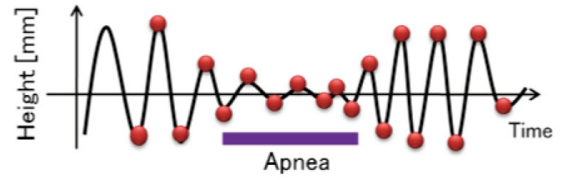

Fig.6 Apnea detection with SI.

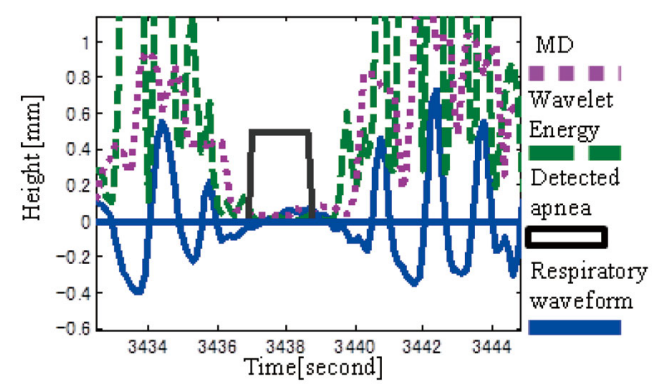

Fig.7 A result using the wavelet-based method.

However, it leads to false positives when the wavelet coefficient is low, depending on the shape of the waveform. We added the maximum displacement criterion to avoid false positives. When the section satisfying this function lasts longer than 1.5 seconds, the section is regarded as apnea. We call this function the "wavelet-based method".

The wavelet-based method commonly detects apneic events in stable and unstable waveforms. However, if the respiratory volume is high around apnea segments, this method fails to detect apnea because of the existence of large frequency energy around the borders between apnea and neighboring respiratory sections (Fig. 7). In addition, newborns tend to deeply breathe in shortly after long apneic events for ventilation. The wavelet-based method can fail to detect borders of long apneic episodes because of the high waveform height. Therefore, we employ a second function whose threshold is stricter.

$\mathrm{MD}<0.1$.

When the section satisfying this function is longer than 1.5 seconds, the section is regarded as apnea. We call this the "MD method". The MD method can detect apnea missed by the wavelet-based method (Fig. 8). This method can detect overall long apnea sections although it can have false negative with high threshold in case of the unstable waveform which contains intermittently high and low values.

The third function is based on SI. If the total duration of units with heights less than $0.1 \mathrm{~mm}$ is more than 1.5 seconds, we regarded the units as apnea (Fig. 6). This function could lead to false positives in the case of shorter-duration apneic events (1.5-2 seconds). To avoid

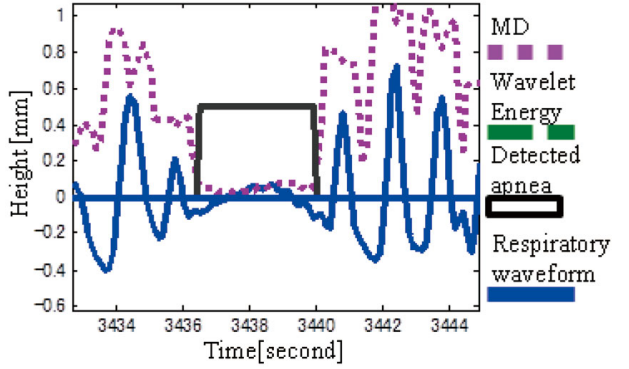

Fig.8 A result using the MD method.

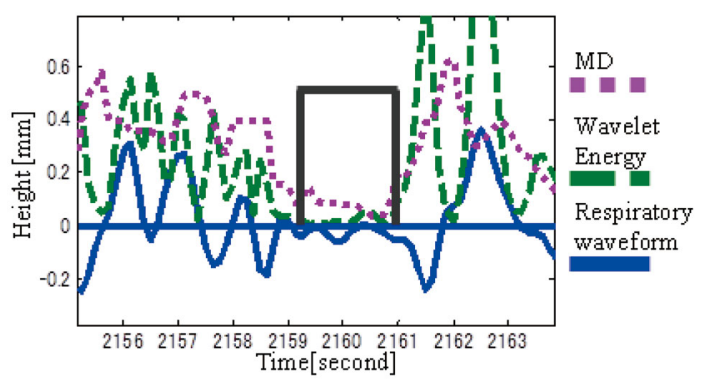

Fig.9 A result using the SI method. The wavelet-base method and the MD method cannot detect the apneic event because the thresholds of these methods are strict to avoid false positive. On the other hand, SI method first detect the position candidate sections of apnea by using etxrema and it can find the candidate section is a real apneic event by using (10), (11) in case of shorter apneic events.

error, we used wavelet energy and MD data to reduce false positives. We regarded the detected shorter sections as apnea when $80 \%$ of the section had a wavelet energy sequence value less than its threshold, or when $50 \%$ of the section had an MD sequence value less than its threshold.

Threshold of wavelet energy $<0.2$,

Threshold of $\mathrm{MD}<0.12$.

The value of SI becomes unstable and yields false positives with long duration when the newborn's respiration is unstable and its wave height is low. To avoid this problem, detected apneic events lasting more than 10 seconds were eliminated. We call this method the "SI method". In shorter-duration apneic events (1.5-2 seconds), the values of wavelet energy and MD tend to exceed their thresholds around the borders between apnea and neighboring respiratory sections because they both use time windows (Fig. 9). To detect shorter apnea, the thresholds of wavelet-based method and MD method should be low, but low threshold causes false positive. The difference of the SI method from the other functions is detecting apneic events by using extrema instead of time window. By using extrema, SI method firstly detects the candidate apneic section. Then, the method 
judges if the shorter sections is apneic event or not. In addition, this method can detect short-duration apneic event that feature values are larger than thresholds of wavelet-based method and MD method in part of the event with equation (10) and (11). Therefore, the SI method mainly detects shorter apneic episodes.

The final result, which we call the apnea label, is a union of the results from these three apnea detection function. We develop three different types of apnea detection function which compensates for each other's weaknesses to ensure that at least one of the three detects apnea, even when thresholds are strict to avoid false positives. Therefore our method can reduce the influence of trade-offs about thresholds between detection accuracy and threshold sensitivity.

\subsection{Experiment}

We have done two experiments. The first one is for the combination of the three different functions. The other one is for the comparison between FFT-power and wavelet energy.

We firstly performed an experiment to evaluate the efficiency of the combination of the three functions. We evaluate the detection accuracy of the three functions and make receiver operating characteristics curves (ROC curve) of them. We performed an experiment using 0.4-1-hour data from the respiratory waveforms of 11 newborns. Before the experiment, we determined our definition of apnea, based on the lectures of a doctor with whom we collaborate as is written in the start of section 4 .

Fig. 10, Fig. 11, and Fig. 12 describe ROC curve showing the relationship between sensitivity and specificity of each apnea detection functions when the following cut-off values are used: wavelet-based method (Wavelet energy 0.01, 0.03, 0.05, 0.07, 0.1 |MD 0.1225), MD method (MD 0.1, 0.15, 0.2, 0.25, 0.3), and SI method ( $\mathrm{H}$ of SI $0.05,0.1,0.2,0.3,0.4$ ). In this experiment, the condition of SI method, "elimination of detected apnea events more than 10s", is invalid for the evaluation of the capability of SI feature. In Fig. 10, the wavelet-based method adopts MD feature for evading false positive and the sensitivity of ROC curve keeps around 0.8. In Fig. 12 , when the condition "elimination of detected apnea events more than $10 \mathrm{~s}$ " is valid, the value of sensitivity becomes low because of the eliminated apnea events more than 10 s and the eliminated unions of the apneic events of false positive. Sensitivity/Spesicifity of SI method is $76.3 \% / 99.8 \%$ when the threshold of $H$ of SI feature is 0.1 .

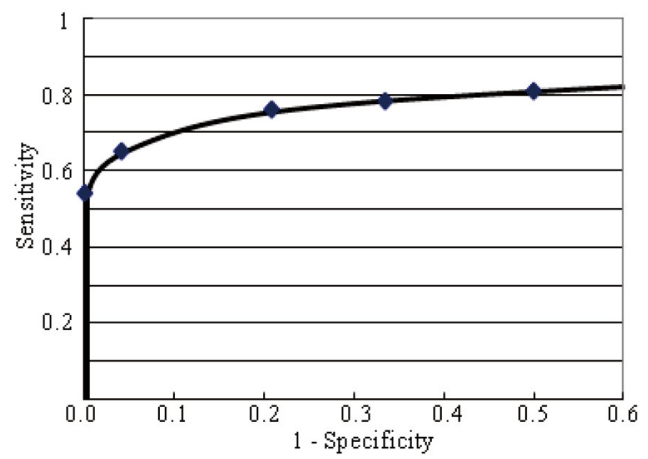

Fig.10 A ROC curve of Wavelet-based method. We change the threshold of wavelet energy: 0.01, 0.03, 0.05, 0.07, 0.1. We fixed the threshold of MD feature: 0.1225 . The value of sensitivity keeps around 0.8 because of the threshold of MD feature.

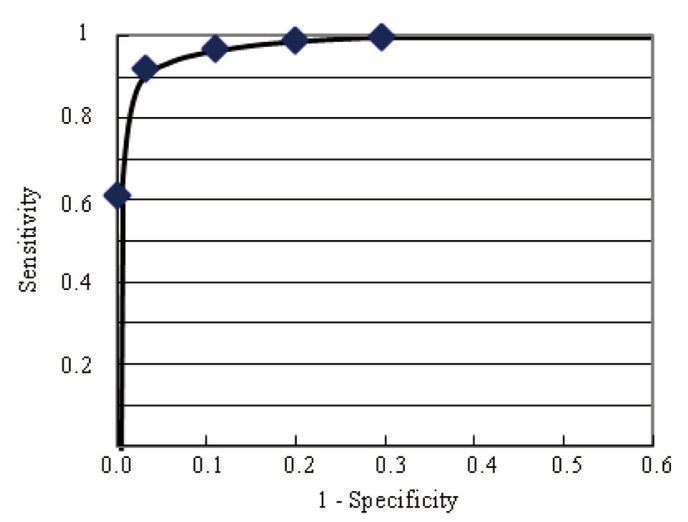

Fig.11 A ROC curve of MD method. We changed the threshold of MD feature: $0.1,0.15,0.2,0.25,0.3$.

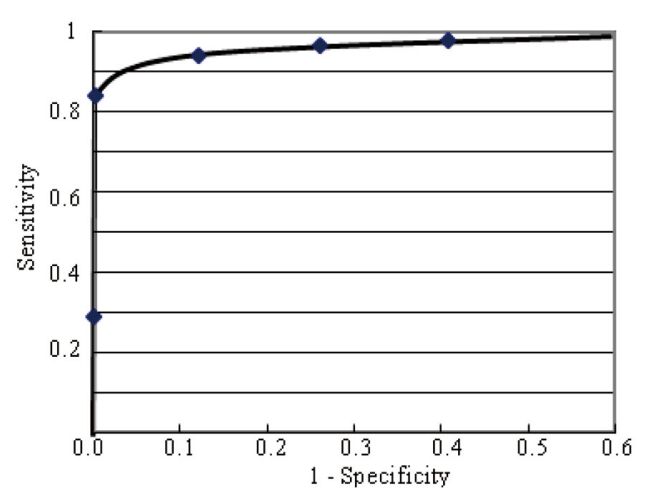

Fig.12 A ROC curve of SI method. We changed the threshold of $\mathrm{H}$ of SI: $0.05,0.1,0.2,0.3,0.4$. The sensitivity of SI method is high, but when the condition "elimination of detected apnea events more than $10 \mathrm{~s}$ ", the value of threshold becomes larger, the value of sensitivity becomes low because of the unions of the sections of false positive.

Table 1 shows the results of our experiment. True positive (TP) means that the method correctly identified apnea. True negative (TN) means that the method correctly identified sections without apnea. False negatives (FN) and TP are expressed in numbers of sections. Time (seconds) is used to express false positives 
Table 1 Accuracy of combination of apnea detection functions.

\begin{tabular}{l|c|c|c|c|c|c}
\hline \hline Method & TP & FN & FP & TN & Sens & Spec \\
\hline \hline Wavelet-based method & 528 & 282 & 13.1 & 28156 & 65.2 & 100 \\
\hline MD method & 494 & 316 & 14.1 & 28155 & 61.0 & 99.9 \\
\hline SI method & 619 & 191 & 69.3 & 28100 & 76.4 & 99.8 \\
\hline $\begin{array}{l}\text { Wavelet-based method } \\
+ \text { MD method }\end{array}$ & 615 & 195 & 25.9 & 28143 & 75.9 & 99.9 \\
\hline $\begin{array}{l}\text { Wavelet-based method } \\
+ \text { MD method } \\
+ \text { SI method }\end{array}$ & 764 & 46 & 84.9 & 28084 & 94.3 & 99.7 \\
\hline \hline
\end{tabular}

TP: True Positive, FN: False Negative, FP: False Positive (seconds), TN: True Negative (seconds), Sens: Sensitivity $=\mathrm{TP} /(\mathrm{TP}+\mathrm{FN}) \times 100(\%)$, Spec: Specificity $=\mathrm{TN} /(\mathrm{TN}+\mathrm{FP}) \times 100(\%)$.

(FP) and TN.

We choice thresholds of the functions to evade false positive, and their sensitivity of the functions are low.

However, our method realizes the high sensitivity of table 1 by combining the three different functions. If respiratory waveform becomes distorted, our method has few false positive, and many true positive.

For the second experiment, we designed a new function to compare apnea detection using FFT versus using wavelets. We used FFT to get the short-time power spectrum within the respiratory frequency band. This power spectrum was designated as FFT-power. We used the Hanning time window of 80 points (10 seconds). The bandwidth of respiratory frequency was set at 0.5 $2.0 \mathrm{~Hz}$ (30-120 cycles/min). FFT was performed at every point (overlapped windows). This step makes an "FFTpower time series". We designed a function for apnea detection using the FFT-based method, which is modified from our method, and compared the results with our standard method. We call this function the FFT approach. The modified part of the function is shown below.

\section{FFT-power $<1.5$,}

$$
\text { Threshold of FFT-power }=14,
$$

where (12) substitutes for (7) in our method and (13) substitutes for (10). We empirically determined thresholds for our method based on a physician's assessment.

We compared the apnea detection accuracy of the FFT approach and our proposed method. We performed an experiment using 0.4-1-hour data from the respiratory waveforms of 11 newborns. Before the experiment, we determined our definition of apnea, based on the lectures of a doctor with whom we collaborate as is written in the start of section 4 . We used the newborns' respiratory waveforms, which were calculated by the FG
Table 2 Accuracy comparison between the FFT approach and our proposed method.

\begin{tabular}{l|l|l|l|l|l|l}
\hline \hline Method & TP & FN & FP & TN & Sens & Spec \\
\hline \hline FFT approach & 736 & 74 & 127.8 & 28041.4 & 90.9 & 99.5 \\
\hline Proposed method & 764 & 46 & 84.9 & 28084.3 & 94.3 & 99.7 \\
\hline \hline
\end{tabular}

TP:True Positive, FN:False Negative, FP:False Positive (seconds), TN: True Negative (seconds), Sens: Sensitivity $=\mathrm{TP} /(\mathrm{TP}+\mathrm{FN}) \times 100(\%)$, Spec: Specificity $=\mathrm{TN} /(\mathrm{TN}+\mathrm{FP}) \times 100(\%)$.

Table 3 Details of the accuracy of FFT approach and proposed method.

\begin{tabular}{l|c|c|c|c|c|c}
\hline \hline \multirow{2}{*}{ Patient } & \multicolumn{7}{|c}{ Results of FFT approach } \\
\cline { 2 - 8 } & TP & FN & FP & TN & Sens & Spec \\
\hline \hline Pal & 30 & 1 & 14.5 & 1455.1 & 96.8 & 99 \\
\hline Pa2 & 48 & 7 & 10.5 & 3298.6 & 87.3 & 99.7 \\
\hline $\mathrm{Pa} 3$ & 25 & 3 & 6.4 & 1090.9 & 89.3 & 99.4 \\
\hline $\mathrm{Pa} 4$ & 69 & 4 & 5.1 & 3668.3 & 94.5 & 99.9 \\
\hline $\mathrm{Pa} 5$ & 118 & 10 & 40.1 & 3319.1 & 92.2 & 98.8 \\
\hline Pa6 & 98 & 9 & 32.4 & 3664.5 & 91.6 & 99.1 \\
\hline Pa7 & 74 & 13 & 3.9 & 3267.6 & 85.1 & 99.9 \\
\hline Pa8 & 8 & 4 & 0 & 3008.4 & 66.7 & 100 \\
\hline Pa9 & 30 & 4 & 3.4 & 1560.1 & 88.2 & 99.8 \\
\hline Pa10 & 17 & 6 & 0 & 1573.4 & 73.9 & 100 \\
\hline Pa11 & 219 & 13 & 11.5 & 2135.4 & 94.4 & 99.5 \\
\hline Total & 736 & 74 & 127.8 & 28041.4 & 90.9 & 99.5 \\
\hline \hline
\end{tabular}

\begin{tabular}{l|c|c|c|c|c|c}
\hline \hline \multirow{2}{*}{ Patient } & \multicolumn{6}{|c}{ Results of Proposed method } \\
\cline { 2 - 7 } & TP & FN & FP & TN & Sens & Spec \\
\hline \hline Pa1 & 31 & 0 & 5.3 & 1464.4 & 100 & 99.6 \\
\hline Pa2 & 52 & 3 & 7.3 & 3301.9 & 94.5 & 99.8 \\
\hline Pa3 & 28 & 0 & 1.9 & 1095.4 & 100 & 99.8 \\
\hline Pa4 & 71 & 2 & 8.6 & 3664.8 & 97.3 & 99.8 \\
\hline Pa5 & 120 & 8 & 16.4 & 3342.9 & 93.8 & 99.5 \\
\hline Pa6 & 102 & 5 & 30.3 & 3666.6 & 95.3 & 99.2 \\
\hline Pa7 & 79 & 8 & 3.9 & 3267.6 & 90.8 & 99.9 \\
\hline Pa8 & 8 & 4 & 0 & 3008.4 & 66.7 & 100 \\
\hline Pa9 & 31 & 3 & 0 & 1563.5 & 91.2 & 100 \\
\hline Pa10 & 18 & 5 & 0 & 1573.4 & 78.3 & 100 \\
\hline Pa11 & 224 & 8 & 11.4 & 2135.5 & 96.6 & 99.5 \\
\hline Total & 764 & 46 & 84.9 & 28084.3 & 94.3 & 99.7 \\
\hline \hline
\end{tabular}

Pa: Patient, TP: True Positive, FN: False Negative, FP: False Positive(seconds), TN: True Negative (seconds), Sens: Sensitivity $=$ TP $/(T P+T N) \times 100(\%)$, Spec: Specificity $=$ $\mathrm{TN} /(\mathrm{TN}+\mathrm{FP}) \times 100(\%)$

vision sensor respiratory monitoring system $^{5)}$.

We empirically determined thresholds of our method on the basis of a doctor's assessment of the results of apnea detection.

Table 2 shows the results of our experiment. Table 3 shows details of Table 2. True positive (TP) means that the method correctly identified apnea. True negative (TN) means that the method correctly identified sections without apnea. False negatives (FN) and TP are expressed in numbers of sections. Time (seconds) is used 


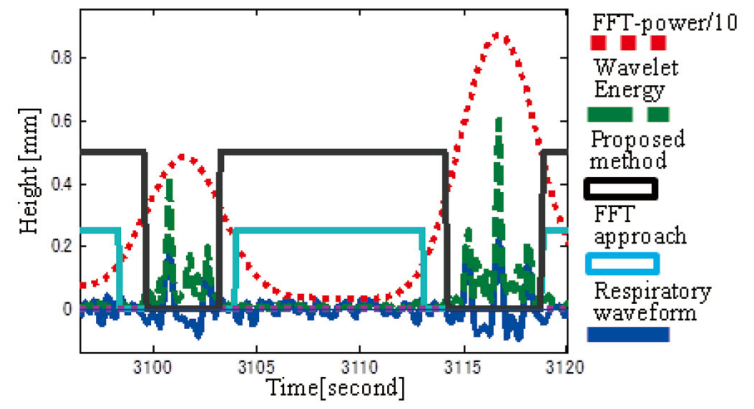

Fig.13 Results of our method and the FFT approach (1). The wavelet-based method and MD method of our method and FFT approach is effective for longer apneic events. However, compared to wavelet, FFT uses longer time window to extract frequency energy and FFT approach tends not to detect borders of longer apneic events.

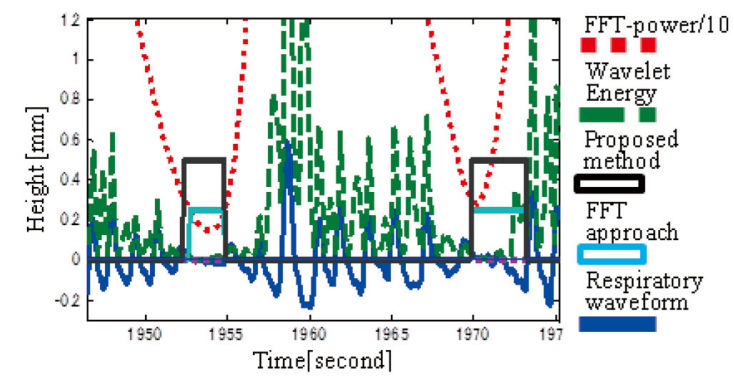

Fig.14 Results of our method and the FFT approach (2). MD method and SI method are effective in shorter apneic events. Wave height of waveform around the apneic events are lower and both our method and FFT approach can detect the apneic events.

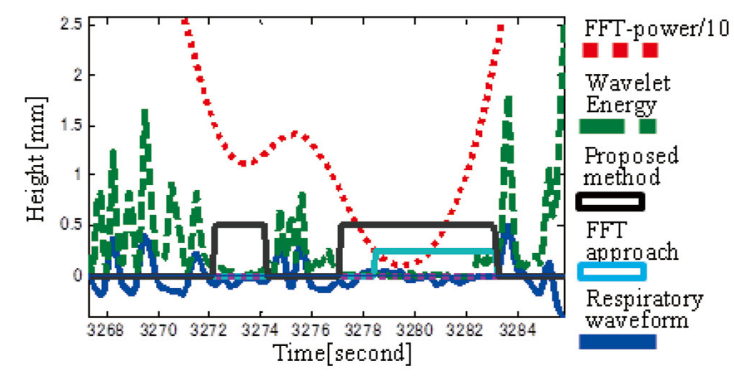

Fig.15 True positive using our method and false negative using the FFT approach. Unlike Fig. 14, FFT method cannot detect the shorter apneic event because wave height of waveform around the apneic event is high. FFT approach tends to be influenced by wave height of waveform around apneic events and FFT-power causes larger trade-offs between detection accuracy and threshold sensitivity than wavelet energy.

to express false positives (FP) and TN.

In the experimental results (Figs. 13-15), the black sections show the results of the proposed method and the teal sections show the results of the FFT approach. We divided the value of the FFT-power by 10 to include it with the other data in a single figure.

Our method and the FFT approach can both detect apnea, including shorter apneic events (1.5-2.0 seconds). Both the wavelet-based method and the MD method detect apnea, especially events lasting more than 3.0 seconds. The SI method especially detects shorter apneic events. The accuracy of these methods is similar but they are different in their details. In Fig. 13, apnea lasting more than 10 seconds in unstable respiration is detected by our method and by the FFT approach (wavelet-based method and MD method). Although these results are regarded as equally correct, the wavelet method can detect overall apnea while the FFT method cannot. In Fig. 14, both our method and the FFT approach detect apnea. The SI method can especially detect shorter apneic events. However, the FFT approach (SI method) cannot detect apnea in the case of Fig. 15 because the FFT-power exceeds its threshold.

These differences are caused by the influence of the time window on both the wavelet and FFT methods. The wavelet method contains less extra frequency energy from the respiratory sections bordering the apnea section than does the FFT method. In the case of the wavelet method, the influence of the extra frequency energy of the respiratory sections corresponds to the width of the wavelet's time window, while the resolution level corresponds to the respiratory frequency. In the FFT method, the influence of extra frequency energy corresponds to the width of the fixed long-duration window, which corresponds to the respiratory frequency band, and the energy value is larger than that of wavelet. This characteristic difference also influences the threshold trade-off.

Our method aims to detect both long and short apneic events overall and robustly. The wavelet method is more appropriate than the FFT method for this purpose.

Most of the false positives and negatives in our method were events lasting less than 2.0 seconds. Fig. 16 shows an example of a false negative resulting from the extra frequency energy of the high wave height around the apnea. In Fig. 17, the several extrema of the distorted waveform caused the left false positive using the SI method. The right false positive using the MD method was caused by the combination of low inspiration and low expiration sections in the waveform. These false positives were generated when waveforms were unstable or had low wave height (0.11-0.12 mm). False negatives and false positives are caused by threshold trade-offs in the wavelet energy and MD functions.

The method of the related work ${ }^{17)}$ regards a section as an apneic event when a respiratory frequency energy dip 


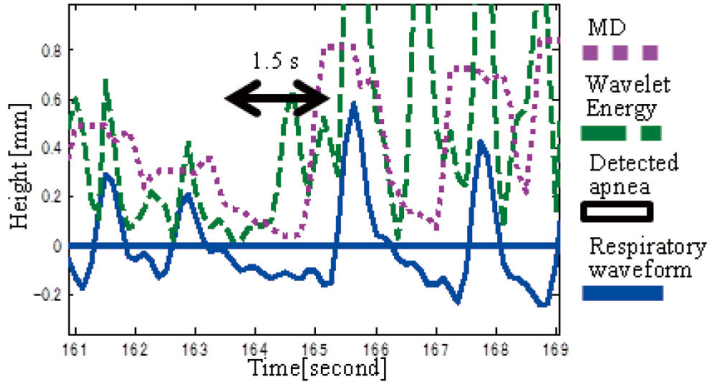

Fig.16 False negative using our method. False negative result due to a large gap in the wave height around the borders between the apnea and respiratory periods. Furthermore, some waveform shapes causes large wavelet energy like upside-down shapes of wavelet.

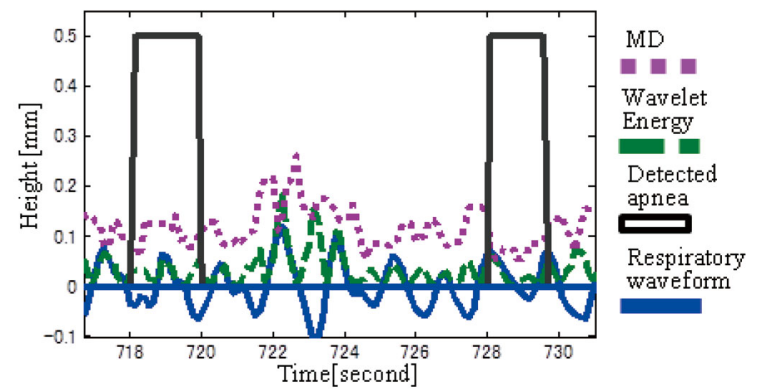

Fig.17 False positive using our method. False-positive results due to low wave height. The left false positive is caused by distorted waveform. The distorted waveform has some extrema. SI method detects small pieces of a breath and regards them as an short apnea period. The right false positive is caused by the combination of low inspiration and low expiration sections in the waveform. In the area, MD value is lower than threshold.

happens which is defined as a fall of more than a threshold value within $30 \mathrm{~s}$. The detected apneic event lasts until patient restart to breath and the frequency energy increases. This method uses FFT to detect apnea periods $\geqq 10$ s even with poor airflow signal quality due to noise.

The disadvantage of FFT is that the value of FFTpower is largely influenced by wave height of waveform in time window. In Fig.15, the values of frequency energy little change in case that there is a little breath at 3275 minutes, which is around comparatively longer apnea periods. The method ${ }^{17)}$ could regard the respiratory section as a part of a long apnea. This case often happens in newborn's waveform. Also, when infrequent respiration lasts, the dip could become quit small and cannot detect apneic events when the events generate.

Respiratory waveform of preterm is instable because varied respiration within a short time and power dips tend to happen in respiratory section. Furthermore, our definition of apnea is that respirations have a wave height less than $0.1 \mathrm{~mm}$ for more than 1.5 seconds. It is difficult to decide the value of parameter for apnea detection. In addition, the time window of FFT is 10 seconds considering frequency resolution whereas the length of apnea is more than 1.5 seconds in preterm newborns. The length of time window could cause the low sensitivity of the method Therefore, only FFT frequency energy is not appropriate for our purpose. If the method uses not only frequency energy but also waveform shape, the method can compensate for the low sensitivity to shorter apnea periods of frequency energy of FFT.

The method of the related work ${ }^{18)}$ detects apnea periods by using half wave, which is a waveform between start of inspiration and end of inspiration. Abnormal respirations (apnea and hypopnea) are characterized by the parameters of the half waves (time, amplitude, and slope). The thresholds for parameters are decided by analysis in each epoch of $30 \mathrm{~s}$ relative to a moving reference interval.

The waveform-shape based method can detect not only longer apnea but also shorter apnea. However, if the values of the waveform show gradual changes, there are few exterma in the waveform and the duration of the half wave becomes longer. The method cannot regard the section as apnea periods. Furthermore, our definition of apnea is that respirations have a wave height less than $0.1 \mathrm{~mm}$ for more than 1.5 seconds. On the other hand, the thresholds of the method are decided from the parameters of half waves in epochs. It is difficult to decide the value of parameter for our apnea definition. In addition, little breath could be distorted by convolution with band-pass filter. Methods related to extrema such as the method of the related work ${ }^{18)}$ and SI method of our method could tend to regard the lowwave height section as apnea. To avoid the results of false positive, the detected apnea periods which are longer than a constant length are eliminated in the both algorithms. The method cannot evaluate the eliminated long sections when the method adopts only waveform shape. Our method uses wavelet energy and MD calculated from a waveform before applying the bandpass filter. Therefore, SI method can detect apnea periods less than $10 \mathrm{~s}$. Wavelet-based method and MD method compensate for SI method by detecting longer apnea periods. 


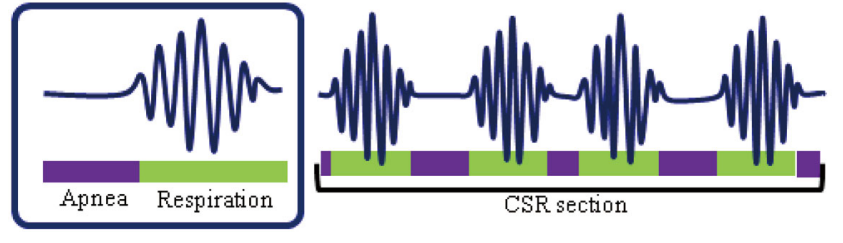

Fig.18 Left: An apnea section and the adjacent respiratory section combine to form one set. Right: Illustration of a CSR section.

\section{CSR Detection}

We developed our CSR detection method using detected apneic events. Our method removes apneic events not related to CSR and regards the remaining apneic events as related to CSR. It then regards the section between neighboring apnea sections as a respiratory section. The apnea section and its adjacent respiratory section are regarded as one set (Fig. 18 Left).

The strict definition of CSR is the presence of at least three consecutive apneic events of at least 3 seconds' duration and each separated by intervals not longer than 20 second $\mathrm{s}^{4}$. Some sections that resemble CSR have respiratory sections lasting more than 20 seconds or apnea sections lasting less than 3 seconds. We aimed to respond flexibly to include not only CSR as strictly defined, but also CSR-like sections. We started with the above definition of CSR and altered it to be more inclusive. We define that CSR section has equal or more than 2 sets and an apneic section which locates in the edge of the CSR section (Fig. 18 Right) considering the strict definition. The respiratory sections of the sets must be equal to or less than 25 seconds and must not include body motion. We empirically see the CSR and CSR-like section tend to have apnea periods more than or equal to 2.5 seconds. When a set unrelated to CSR is removed, the remaining apnea sections become part of the candidate CSR section. At the boundary of a CSR section, there is a set with a longer respiratory section. Our method utilizes this phenomenon for accurately detecting a CSR section in time axis. To detect CSR sections, we mainly used the spurious periodicity feature (SPF), which indicates the periodicity of apneic events.

\subsection{Proposed Method}

During preprocessing, we removed the sets that had respiratory sections lasting more than 25 seconds or body motion and regarded the remaining sets as candidate CSR sections. At this step, we could not distinguish the correct CSR sections from the candidate CSR sections (Fig. 19). Next, we removed the sets unrelated to CSR from the candidate CSR sections,
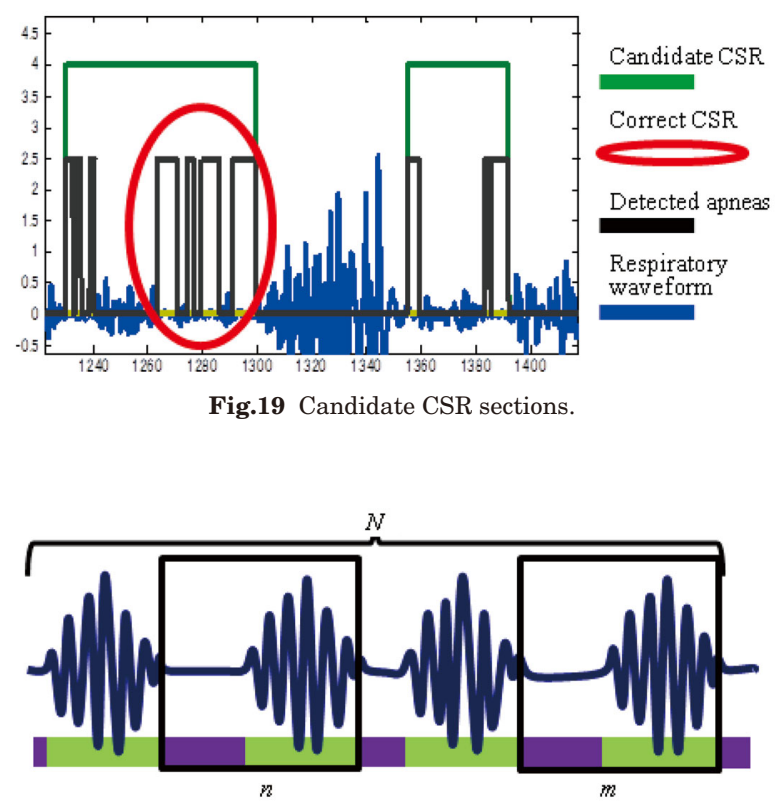

Fig.20 Illustration of SPF calculation.

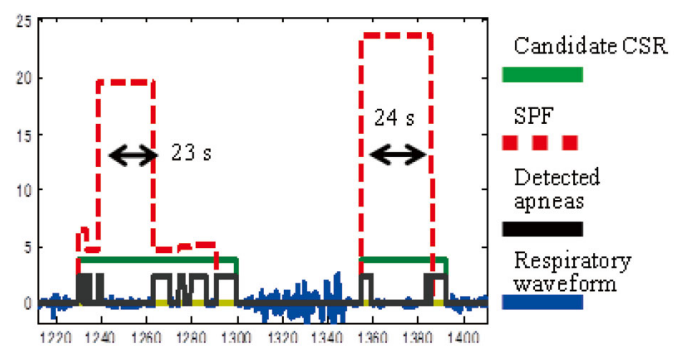

Fig.21 SPF values of each set.

primarily using SPF.

We calculated the SPF for every set within each candidate CSR section. The SPF value is a mean of the absolute values of the finite difference between the length of the respiratory section of interest and that of other respiratory sections (Fig. 20). The length of the sets tends to be similar to that of the respiratory section in a CSR. If the set does not belong to a CSR section, the SPF of the set becomes larger because of the length discrepancy. Fig. 21 shows the results of an SPF calculation. The SPF becomes large if the set is not related to CSR and its respiratory section is long.

$$
S P F_{m}=\frac{1}{N-1} \sum_{n \neq m}^{N}\left|L_{n}-L_{m}\right|,
$$

where $N$ is the number of sets of a candidate CSR section and $L_{n}$ and $L_{m}$ are the lengths of the respiratory sections of the $n$-th and $m$-th sets from the left, respectively.

We focused on the sets with longer respiratory sections to detect the boundaries of CSR sections. The respiratory section of the boundary set is often longer and the SPF increases. If the focus set satisfies the following functions, the set is regarded as unrelated to 


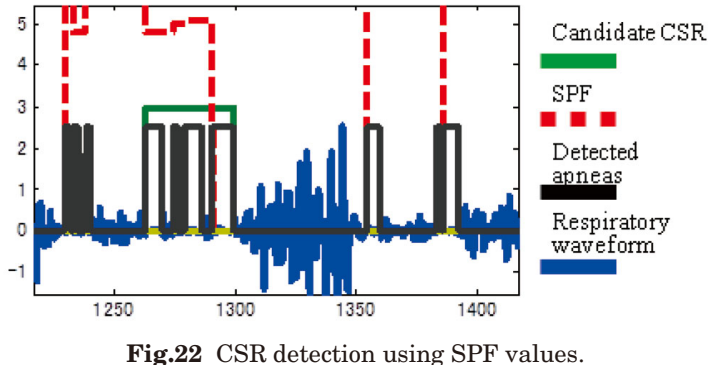

CSR.

$$
\begin{aligned}
& L_{n}>15 \mathrm{~s} \text { and } S P F>18 \mathrm{~s}, \\
& L_{n}>15 \mathrm{~s} \text { and } S P F / L_{n}>0.7 .
\end{aligned}
$$

The value of $S P F / L_{m}$ becomes larger when the respiratory section of the set is much longer than the others, even in the case of an SPF less than 18 seconds.

After removing those sections unrelated to CSR, both longer and shorter candidate CSR sections remain. The shorter sections sometimes consist mostly of sets that have respiratory sections less than 3 seconds. These sections are possible false positives. We adopted the following functions to avoid false positive CSR and CSRlike sections:

In the case of Num $=3$ : If all three apneic events last more than 2.5 seconds, the candidate section is regarded as CSR (17);

In the case of Num $>3$ and Num < 10 : If half of the apneic events last more than 2.5 seconds, the candidate section is regarded as CSR (18),

where Num is the number of apnea sections in the candidate CSR section. Fig. 22 shows the results of our method. Our method successfully detected the CSR section.

\subsection{Experiment}

We performed an experiment using 0.4-1-hour data from the respiratory waveforms of nine newborns with CSR.

Table 4 shows the results of the experiment. True positive (TP) means that the method correctly identified CSR. TP, $\mathrm{FN}$, and FP are expressed as number of sections.

The SPF can help determine whether sets with respiratory sections lasting more than 20 seconds belong to CSR or not. Fig. 23 shows an example of CSR including sets with respiratory sections lasting more than 20 seconds. The SPFs of the sets located in the central area are comparatively low. Although the CSR section has two sets with respiratory sections of 20-25 seconds, our method can determine that they are CSR. Our method avoids false positives in the case of Fig. 24, where CSR does not include respiratory sections longer than 20
Table 4 Accuracy of our method.

\begin{tabular}{l|l|l|l}
\hline \hline Method & TP & FN & FP \\
\hline \hline Our method & 45 & 1 & 0 \\
\hline \hline
\end{tabular}

TP: True Positive, FN: False Negative, FP: False Positive (number)

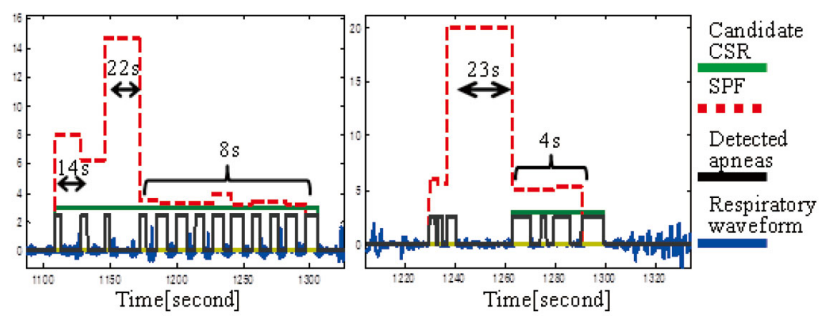

Fig.23 (Left) CSR including respiratory sections more than 20 seconds.

Fig.24 (Right) CSR not including respiratory sections more than 20 seconds.

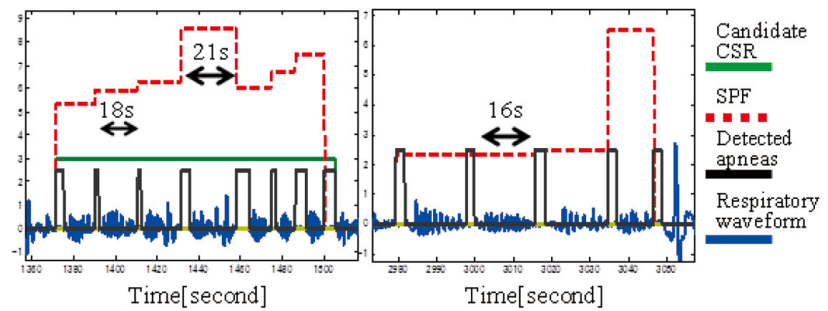

Fig.25 (Left) CSR including a respiratory section less than 3 seconds.

Fig.26 (Right) An example of a false negative using our method.

seconds. Furthermore, our method can detect not only CSR but also CSR-like sections, including respiratory sections lasting less than 3 seconds (Fig. 25).

The use of $S P F / L_{m}$ avoids false positives in cases such as that shown in Fig. 24. In the near case, although one of the sets has a significantly larger value than the others, the entire respiratory section is less than 20 seconds, and the value of SPF is less than 18 seconds. The value of $S P F / L_{m}$ exceeds its threshold because the value of SPF is close to $L_{m}$.

Fig. 26 shows the false negative obtained with our method. In Fig. 26, most apnea sections are less than 2.5 seconds. Although the waveform shows cyclical and gradual breath strength change between apnea and normal breath, our method cannot detect the CSR section because of the strict thresholds to avoid false positives.

Our method did not result in false positives in this experiment. This is because apneic events lasting more than 2.5 seconds were often present in the CSR sections. In the other sections, there were few apneic events lasting more than 2.5 seconds and separated by 
intervals less than 20 seconds in local regions. Apneic events lasting less than 2.5 seconds often arise in these areas. In this case, functions (15) and (16) can remove the candidate CSR sections, which include few apneic events lasting more than 2.5 seconds and comparatively numerous apneic events under 2.5 seconds.

We tried to detect CSR sections using only information about the length of the respiratory sections. Our method can detect CSR sections in the waveforms of neonates. The use of the SPF contributes to the detection procedure. However, our method cannot detect a CSR section if most apneic events last less than 2.5 seconds (Fig. 26).

\section{Conclusion}

In this paper, we propose an apnea detection method and a method to detect and estimate the severity of CSR.

The sensitivity of apnea detection of our method is $94.3 \%$, and its specificity is $99.7 \%$. These good results come from combining the wavelet-based method, the MD method, and the SI method. The SI method can mainly detect shorter episodes of apnea (1.5-2.0 seconds). Using the SI method, we can keep the thresholds of the wavelet-based method and the MD method lower, and these methods can detect comparatively long apneas robustly. Our method provides sufficient results to be used for CSR detection.

There are two reasons why we based our CSR detection method on respiratory sections between apneic events. The first is that the respiratory waveforms of infants are often unstable. Secondly, traditional methods of CSR detection based on frequency of the envelope curve of the waveform are not valid for CSR detection in infants. Although our method can detect CSR sections accurately along the time axis, it tends to result in false negatives if most apneic events last less than 2.5 seconds. Incorporating additional features related to the shape of the waveform would result in more accurate detection of CSR.

A CSR detection method based on apnea could be used to extract features of CSR, such as duration of apnea and length of respiratory sections. We believe that our method will help in the development of a respiratory function evaluation system for newborns.

\section{References}

1) Di Fiore JM: "Neonatal cardiorespiratory monitoring techniques", Seminars in Neonatology, 9, 3, pp.195-203 (2004)

2) D. Takahashi, T. Hiroma, M. Matsui, H. Dainobu, M. Inoue and T. Nakamura: "Investigation of the Usefulness of Lightweight
Mainstream Capnometer with a Very Low Dead Space Volume", Journal of Japan Society of Perinatal and Neonatal Medicine, 45, 4, pp.1078-1082 (2009)

3) H. Rigatto and J.P. Brady: "Periodic Breathing and Apnea in Preterm Infants. II. Hypoxia as a Primary Event", Pediatrics, 50, 2, pp.219-228 (1972)

4) A.J. Oliveira, M.L. Nunes, A. Fojo-Olmos, F.M. Reis and J.C. da Costa: "Clinical Correlates of Periodic Breathing in Neonatal Polysomnography", International Federation of Clinical Neurophysiology, 15, 10, pp.2247-2251 (2004)

5) D.H. Kelly and D.C. Shannon: "Periodic Breathing in Infants with Near-Miss Sudden Infant Death Syndrome", Pediatrics, 63, 3, pp.355-360 (1979)

6) D.C. Shannon, D.W. Carley and D.H. Kelly: "Periodic Breathing: Quantitative Analyses and Clinical Description", Pediatric Pulmonology, 4, 2, pp.98-102 (1988)

7) Y. Kurami, Y. Ito, M. Natori, K. Ohzeki and Y. Aoki: "Development of Non-contact Respiratory Monitoring System for Newborn Using a FG Vision Sensor", IEEJ Trans., 130, 9, pp.1581-1587 (2011)

8) K. Nakazawa, M. Nakajima and H. Kobayashi: "Development of 3D Shape Measurement System Using Fiber Grating", IEICE Trans. Inf. \& Syst., J-69-D, 12, pp.1929-1935 (1986)

9) A. Garde, B.F. Giraldo, R. Jane, I. Diaz, S. Herrer, S. Benito, M. Domingo and A. Bayes-Genis: "Characterization of Periodic and Non-Periodic Breathing Pattern in Chronic Heart Failure Patients", EMBS 2008 30th Annual International Conference of the IEEE, pp.3227-3230 (2008)

10) A. Garde, L. Sornmo, R. Jane and B.F. Giraldo: "Breathing Pattern Characterization in Chronic Heart Failure Patients Using the Respiratory Flow Signal", Biomedical Engineering, 38, 12, pp.3572-3580 (2011)

11) N.N. Finer, R. Higgines, J. Kattwinkel and R.J. Martin: "Summary Proceedings from the Apnea-of-Prematurity Group", Official Journal of The American Academy of Pediatrics, 117, 3, pp.S47S51 (2006)

12) Standards of Practice Committee of the American Sleep Disorders Association: "Practice Parameters for the Indications for Polysomnography and Related Procedures", Standards of Practice Committee of the American Sleep Disorders Association, 20, pp.406-422 (1997)

13) P. Varady, T. Micsik, S. Benedek and Z. Benyo: "A Novel Method for the Detection of Apnea and Hypopnea Events in Respiration Signals", IEEE Transactions on Biomedical Engineering, 49, 9, pp.936-942 (2002)

14) J.Y. Tian and J.Q. Liu: "Apnea Detection Based on Time Delay Neural Network", Engineering in Medicine and Biology 27th Annual Conference, pp.2571-2574 (2005)

15) O. Fontenla-Romero, B. Guijarro-Berdin as, A. Alonso-Betanzos and V. Moret-Bonillo: "A New Method for Sleep Apnea Classification Using Wavelets and Feedforward Neural Networks", Artificial Intelligence in Medicine, 34, 1, pp.65-76 (2005)

16) H. Lee, C.G. Rusin, D.E. Lake, M.T. Clark, L. Guin, T.J. Smoot, A.O. Paget-Brouwn, B.D. Vergales, J. Kattwinkel, J.R. Moorman and J.B. Delos: "A New Algorithm for Detecting Central Apnea in Neonates", Physiological Measurement, 33, 1, pp.1-17 (2012)

17) H. Nakano, T. Tanigawa, T. Furukawa and S. Nishima: "Automatic Detection of Sleep- Disordered Breathing from a Single-Channel Airflow Record", European Respiratory Journal, 29, 4, pp.728-736 (Aug. 2007)

18) P.J. van Houdt, P.P. Ossenblock, M.G. van Erp, K.E. Schreuder, R.J. Krijn, P.A. Boon and P.J. Cluitmans, "Automatic Breath-toBreath Analysis of Nocturnal Polysomnographic Recordings", International Federation for Medical and Biological Engineering, 49, 7, pp.819-830 (2011)

19) I. Daubechies and W. Sweldens, "Factoring Wavelet Transforms into Lifting Schemes", The J. of Fourier Analysis and Applications, 4, 3, pp.247-269 (1998) 


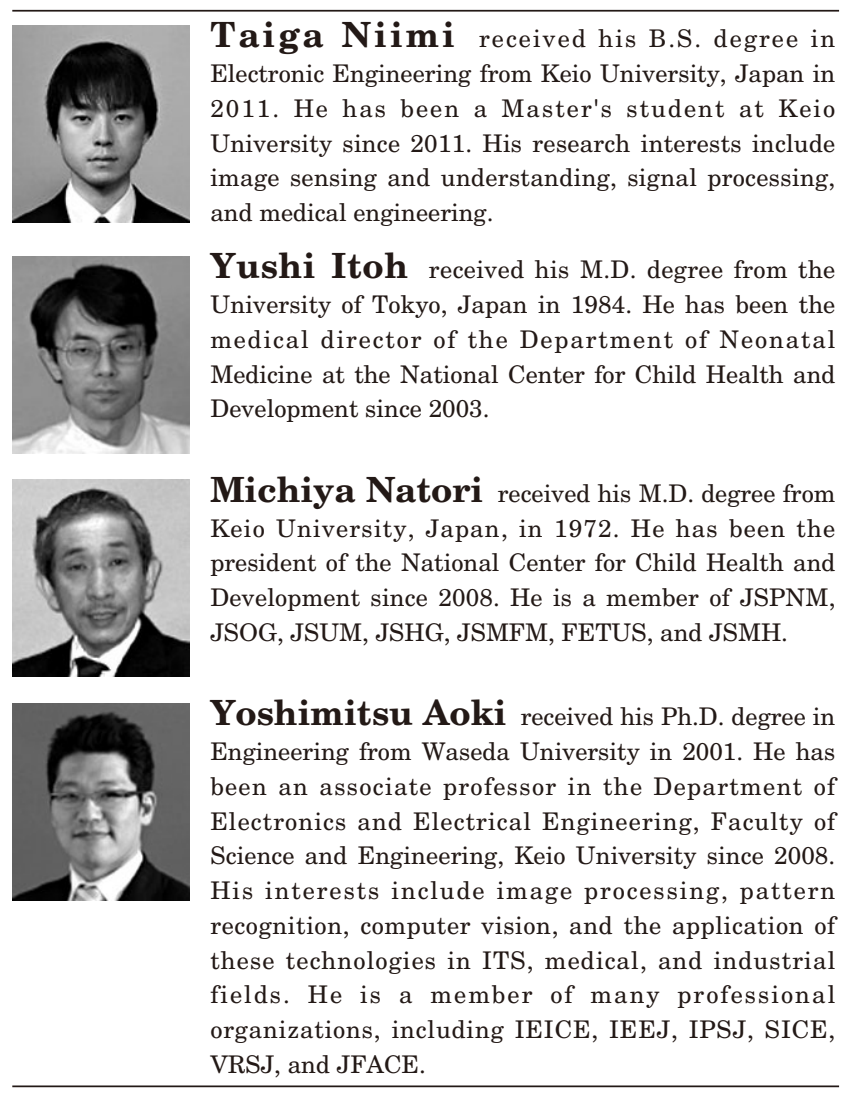

